\title{
Indentifying Risk Factor for Low out Come in General Surgical Practice Using Portsmouth Possum Scoring System
}

\author{
${ }^{1}$ Dr M Vennila MS, ${ }^{2}$ Dr K Santhanalakshmi MS, \\ ${ }^{I}$ Junior Resident, Department of General Surgery, Govt Medical College \& ESI Hospital, \\ Varatharajapuram, Coimbatore \\ ${ }^{2}$ Assistant Professor, Department of General Surgery, Govt Theni Medical College \& Hospital, Theni
}

\section{Introduction}

The basic aim of any surgical procedure is to reduce in morbidity and mortality rates. By this scoring system, comparing the influence on adverse outcome and also assess the efficiency of that particular procedure and their by provide the quality of care. The risk of post operative morbidity and mortality was predicted by using several scoring system. These scoring system can used for several surgical procedure and also qualitative assessments of different surgeons, hospital and countries possum (physiological and But comparison using crude morbidity and mortality rates is fallacious, because of differences in general health of the local population and variable presentation of the patient"s condition. Risk scoring seeks to quantify a patient"s risk of adverse outcome based on the severity of illness derived from data available at an early stage of the hospital stay.

The possible outcome of a surgical operation must be determined to cause evolution of more effective treatment regimens. Therefore, there is a need for an accurate risk adjusted scoring system, which should be specific to the patient being studied, should incorporate the influence of the diagnosis for which he is being subjected for surgery, whether elective or emergency and allow for assessment of variable presentation of each patient, to allow assessment of the efficiency of the particular procedure performed. It should also, be easy to use, fast, and comparable among different patient groups. Such a scoring system would allow for comparison of quality of care provided. It could be used to help set a benchmark acceptable adverse outcome rate for a particular procedure, by comparing the mortality rates among different surgeons. It would also allow for comparison of efficacy of various procedures by comparing the differences in observed to expected mortality rates. It would result in a better and meaningful surgical audit and also help in faster adaptation of a new procedure by comparing the reduction in the observed to expected adverse outcome rate. It could be used in predicting the individual patient"s prognosis, influence treatment decisions and help in rationalising regimens.

The Physiological and Operative Severity Scoring system for the enumeration of Morbidity and mortality (POSSUM) has been proposed as a risk adjusted scoring system to allow for direct comparison between the observed and expected adverse outcome rates. It has been called as a surgeon based scoring system. The Portsmouth POSSUM is a modification of the POSSUM scoring system, incorporating the same variables and grading system, but a different equation, which provides a better fit to the observed mortality rate, which is an important and objective measure of outcome. It has already found use in general, vascular, colorectal, oesophageal and laparoscopic procedures but the studies mostly involved patients in developed countries, where the patient characteristics, presentation and available resources differ from our setup.

Hence, there is a need to test the validity of P-POSSUM scoring system in the Indian scenario where malnourishment is a common problem, presentation frequently delayed and resources limited, all of which can influence the patient"s complication rate, even with adequate quality of care provided. Hence, the scoring system should be able to incorporate these factors to predict an accurate mortality rate. The PPOSSUM scoring system, which includes both physiological and operative finding parameters, has been proposed to address these concerns. Therefore, there is a need to test whether the P- POSSUM scoring system is able to effectively address these concerns while arriving at the expected mortality rate in the Indian scenario. Major surgeries (elective and emergency), as defined by the POSSUM scoring system, constitute the important high risk group of patients where, the comparison of observed to expected mortality rate would be expected to yield significant results and, determination of the possible causes for the adverse outcome in patients who succumb following the surgical procedure, would be more beneficial. This study was undertaken to assess the validity of P-POSSUM scoring system in patients undergoing major surgeries in our setup and, to try to analyse the causes for low outcome in this high risk group.

\section{Aims and Objectives:}

1) To assess the validity of Portsmouth POSSUM scoring system in predicting anticipated mortality rate and to compare with the actual mortality rate in general surgical patients admitted for major surgical procedure.

2) To assess validity of Portsmouth POSSUM scoring system in identifying risk factors for adverse outcome. 


\section{Source of data:}

\section{Methodology}

This prospective study was carried out on patients undergoing major general surgical procedures admitted in the Department of General Surgery of Government Rajaji Hospital, Madurai Medical College, Madurai

\section{Study period:}

The study period was from NOVEMBER 2012 to OCTOBER 2013 and the period of follow up was 30 days following the surgical procedure.

\section{Method of collection of data:}

Patients admitted under general surgery and scheduled to undergo major surgical procedures were scored according to their physiological and operative findings using a proforma sheet

\section{Inclusion criteria:}

Patients undergoing any of the following major surgical procedures as defined by the POSSUM scoring system,

1. Any laparotomy

2. Cholecystectomy with choledochotomy

3. Bowel resection

4. Major amputation

5. Peripheral vascular procedure

\section{Exclusion criteria:}

1. Age less than 12 years

2. Day care surgery

3. Follow up period criteria not met.

4. All minor, moderate, major+ surgeries as defined by POSSUM scoring systems.

Patients were informed regarding the aims and objectives of study and a detailed informed written consent was taken prior to inclusion into the study. The study protocol was approved by the local ethical clearance committee of this hospital. During hospitalisation relevant history was collected and appropriate investigations as deemed necessary were done using standard procedures. The patients were then scored depending on their physiological parameters and the intra operative findings were noted and a final expected mortality rate was calculated

\section{Portsmouth Physiological And Operative Severity Score For The Enumeration Of Mortality And} Morbidity (P-Possum) Physiological Scoring

\begin{tabular}{|c|c|c|c|c|}
\hline & 1 & 2 & 4 & 8 \\
\hline Age & $<60 \mathrm{yrs}$ & $61-70 y r s$ & $>70 \mathrm{yrs}$ & \\
\hline Cardiac sgns & No failure & $\begin{array}{l}\text { Diuretic, } \\
\text { Antianginal, } \\
\text { Digoxin or } \\
\text { Anti } \\
\text { hypertensive } \\
\text { therapy }\end{array}$ & $\begin{array}{l}\text { Peripheral edema, } \\
\text { Warfarin therapy }\end{array}$ & Raised JVP \\
\hline Chest $\mathrm{X}$ ray & & & $\begin{array}{l}\text { Borderline } \\
\text { cardiomegaly }\end{array}$ & Cardiomegaly \\
\hline $\begin{array}{l}\text { Respiratory } \\
\text { History }\end{array}$ & No dyspnoea & $\begin{array}{l}\text { Dyspnoea on } \\
\text { exer }\end{array}$ & Limiting dyspn & $\begin{array}{l}\text { Dyspnoea at } \\
\text { rest (rate } \\
>30 / \mathrm{min} \text { ) }\end{array}$ \\
\hline Chest $\mathrm{x}$ ray & & Mild COAD & Moderate COAD & $\begin{array}{l}\text { Fibrosis or } \\
\text { consolitation }\end{array}$ \\
\hline $\begin{array}{l}\text { Blood Pressure } \\
\text { (systolic) (mm } \\
\text { of } \mathrm{Hg})\end{array}$ & $110-130$ & $\begin{array}{l}131-170 \\
100-109\end{array}$ & $\begin{array}{l}>171 \\
90-99\end{array}$ & $<89$ \\
\hline $\begin{array}{l}\text { Glasgow coma } \\
\text { scale }\end{array}$ & 15 & $12-14$ & $9-11$ & $<8$ \\
\hline $\begin{array}{l}\text { Pulse Rate } \\
\text { (beats/mt) }\end{array}$ & $50-80$ & $81-10040-49$ & $101-120$ & $>121<39$ \\
\hline $\begin{array}{l}\text { Haemoglobin } \\
(\mathrm{g} / \mathrm{dl})\end{array}$ & $13-16$ & $\begin{array}{c}11.5-12.9 \\
16.1-17\end{array}$ & $\begin{array}{l}10-11.4 \\
17.1-18\end{array}$ & $\begin{array}{l}<9.9 \\
>18.1\end{array}$ \\
\hline White cell count & $4-10$ & $10.1-20$ & $>20.1<3.1$ & \\
\hline
\end{tabular}


Indentifying Risk Factor for Low out Come in General Surgical Practice Using...

\begin{tabular}{|l|l|l|l|l|}
\hline$\left(\mathrm{X} 10^{12} / \mathrm{l}\right)$ & & $3.1-4$ & & \\
\hline Urea(mmol/1) & $<7.5$ & $7.6-10$ & $10.1-15$ & $>15.1$ \\
\hline $\begin{array}{l}\text { Sodium } \\
(\mathrm{mmol} / \mathrm{l})\end{array}$ & $>136$ & $131-135$ & $126-130$ & $<125$ \\
\hline $\begin{array}{l}\text { Potassium } \\
(\mathrm{mmol} / \mathrm{l})\end{array}$ & $3.5-5$ & $3.2-3.4$ & $\begin{array}{l}2.9-3.1 \\
5.4-5.9\end{array}$ & $\begin{array}{l}\text { Atrial fibrillation } \\
\text { (rate 60-90) }\end{array}$ \\
\hline ECG & Normal & $5.2-5.3$ & $\begin{array}{l}\text { Any abnormal } \\
\text { rhythm or } \\
5\end{array}$ \\
& & & $\begin{array}{l}\text { ectopics/min, } \\
\text { Q waves or } \\
\text { ST/T wave } \\
\text { changes }\end{array}$ \\
\hline
\end{tabular}

\begin{tabular}{|c|c|c|c|c|}
\hline \multicolumn{5}{|c|}{ Operative scoring } \\
\hline & 1 & 2 & 4 & 5 \\
\hline Operative severity & Minor & Moderate & Major & Major + \\
\hline $\begin{array}{l}\text { Multiple } \\
\text { procedures }\end{array}$ & 1 & & 2 & $>2$ \\
\hline $\begin{array}{l}\text { Total blood } \\
\operatorname{loss}(\mathrm{ml})\end{array}$ & $<100 \mathrm{ml}$ & $100-500 \mathrm{ml}$ & $501-1000 \mathrm{ml}$ & $>1000 \mathrm{ml}$ \\
\hline Peritoneal soiling & None & $\begin{array}{l}\text { Minor(serous } \\
\text { fluid) }\end{array}$ & Local pus & $\begin{array}{c}\text { Free bowel } \\
\text { content,pus or blood }\end{array}$ \\
\hline $\begin{array}{l}\text { Presence of } \\
\text { malignancy }\end{array}$ & None & Primary only & Nodal metastases & Distant metastases \\
\hline Mode of surgery & Elective & & $\begin{array}{l}\text { Emergency } \\
\text { resuscitation of } \\
>2 \mathrm{~h} \text { possible, } \\
\text { Operation }<24 \mathrm{~h} \\
\text { after admission }\end{array}$ & $\begin{array}{c}\text { Emergency } \\
\text { (immediate surgery) } \\
<2 \text { h needed }\end{array}$ \\
\hline
\end{tabular}

Physiological score (12-88), Operative score $(\mathbf{9 - 4 4})$

For mortality it is,

Loge $[R / 1-R]=(0.1692 \times P S)+(0.155 \times$ OS $)-9.065$.

Where $\mathrm{R}=$ risk of mortality 8 .

The patients were then followed up for a period of 30 days following the surgical procedure and complications if any, were noted depending upon the following criteria as defined for POSSUM scoring systems.

\section{Wound haemorrhage:}

Local haematoma requiring evacuation.

Deep haematoma:

Postoperative bleeding requiring re-exploration.

\section{Chest infection:}

Production of purulent sputum with positive bacteriological cultures, with or without chest radiography changes or pyrexia, or consolidation seen on chest radiograph.

Wound infection:

Wound cellulitis or the discharge of purulent exudate.

Urinary infecion

The presence of $>105$ bacteria/ml with the presence of white cells in the urine, in previously clear urine.

Deep infection:

The presence of an intra-abdominal collection confirmed clinically or radiologically.

Septicaemia:

Positive blood culture.

Pyrexia of unknown origin:

Any temperature above 370 Celsius for more than 24 hours after the original pyrexia following surgery (if present) had settled, for which no obvious cause could be found.

Wound dehiscence:

Superficial or deep wound breakdown.

Deep venous thrombosis and pulmonary embolus:

When suspected, confirmed radiologically by venography or ventilation/perfusion scanning, or diagnosed at post mortem.

Cardiac failure: 
Symptoms or signs of left ventricular or congestive cardiac failure, which required alteration from preoperative therapeutic measures.

\section{Impaired renal function:}

Arbitrarily defined as increase in blood urea $>5 \mathrm{mmol} / \mathrm{l}$ from preoperative levels.

Hypotension:

A fall in systolic blood pressure below $90 \mathrm{mmHg}$ for more than 2 hours as determined by sphygmomanometry or arterial pressure transducer measurement.

\section{Respiratory failure:}

Respiratory difficulty requiring emergency ventilation.

Anastomotic leak:

Discharge of bowel content via the drain, wound or abnormal orifice.

Statistical methods:

The expected mortality rate was obtained using linear regression analysis and the O: E ratio was calculated. Chi square test was then applied to obtain the $\mathrm{p}$ value to note any significant difference between the predicted death rate and the actual outcome. Rate of increment in deaths for each risk factor was calculated based on the hypothesis that deaths were linearly related with the score for each of the studied risk factors and,,$t^{\prime \prime}$ test was applied to validate this hypothesis.

\section{Results}

A total of seventy five patient undergoing major surgery in government Rajaji hospital during the period of November 2012 to October 2013 were included in the study protocol. Twenty patients underwent two major surgical operations. There were 55emergency and 20 elective procedures.

Mode of surgery:

There were 55 emergency and 20 elective surgeries performed.

\begin{tabular}{|l|l|}
\hline Elective & $\mathbf{2 6 . 6 \%}$ \\
\hline Emergency & $\mathbf{7 3 . 3 \%}$ \\
\hline
\end{tabular}

\section{Types of major surgeries performed:}

There were four types of major surgeries performed in our group, there are laporotomy, resection anastomosis, major amputation and cholecystectomy.

\section{Outcome of surgery:}

Of the 75 procedures studied, 12 of them were associated with death of the patient resulting in crude mortality rate of $16 \%$

\section{Observed: Expected mortality rate:}

Comparison of observed and P-POSSUM predicted mortality rates was done using linear analysis represented in table 2 and graph 5. An observed to expected ratio (O: E) of 0.96 was obtained and there was no significant difference between the predicted and observed values $(\mathrm{P}=0.048)$.

\section{Mode of surgery:}

There were 3 deaths (26.6\%) among 20 elective cases (15\%) and 9deaths (73.3\%) from 55 emergency major surgeries $(85 \%)$ in our study. A positive rate of increment of deaths per score was obtained.

\begin{tabular}{|l|c|c|c|}
\hline Mode Of Surgery & No Of Cases & No Of Cases Dead & No Of Cases Alive \\
\hline Elective & 20 & 3 & 17 \\
\hline Emergency & 55 & 9 & 46 \\
\hline
\end{tabular}

\section{Malignancy:}

There were 3 cases with malignancies on which surgery was done. They are with primary only, without lymph node involvement, accounting forl deaths. A positive rate of increment of deaths per score was obtained suggesting association of malignancy with adverse outcome and statistically significant association was obtained.

\section{Electrocardiogram findings:}

There were 30 cases with electrocardiographic abnormalities (scored 4 points) who were subjected to major general surgery and all 5 patients died. A positive rate of increment of deaths with score was obtained. 


\section{Peritoneal contamination:}

In a total of 65 surgeries, some degree of peritoneal contamination was found and 10 surgeries (13\%) were associated with free bowel content, blood or gross pus. A positive rate of increment of deaths per score was obtained suggesting association of degree of peritoneal contamination with adverse outcome but was not found to be statistically significant.

\section{Total blood loss:}

In our study we found majority of cases resulted in $100-200 \mathrm{ml}$ blood loss (61 cases, $81 \%$ ), which also accounted for majority of mortalities (10 cases, $13 \%$ ).There were 14 cases with $500-1000 \mathrm{ml}$ blood loss of which 2 case died during the study period. There were no cases with $>1000 \mathrm{ml}$ blood loss in our study. On analysis, a positive rate of increment with deaths in relation to increase in scores was found, suggesting correlation of higher blood loss with more adverse outcome and was found to be statistically significant.

\section{Serum potassium :}

Our study group comprised of 75 surgeries performed on patients with some degree of imbalance in serum potassium concentration which accounted for 12 deaths (16\%). On analysis a positive rate of increment per score was obtained suggesting correlation of deaths with scoring of imbalance in potassium concentration but was not statistically significant.

\section{Serum Sodium:}

Surgeries done on cases with serum sodium abnormalities accounted for 75 cases with mortality occurring in 12 cases (16\%). A positive rate of increment of deaths was found on analysis and was found to be statistically significant.

\section{Blood urea:}

A total of 20 procedures $(27 \%)$ were performed on patients with elevated blood urea levels and these cases accounted for 3 deaths (15\%)q with the majority of deaths occurring in the highest score group A positive rate of increment of death wit score was obtained and was found to be statistically significant.

\section{White cell count:}

Surgeries done on patients with leucocytosis accounted for 75 cases 12 deaths (16\%) occurring in this group. A positive rate of increment of deaths with higher score was obtained and was not found to be statistically significant.

\section{Haemoglobin:}

A majority of the procedures were done on patients with abnormalities in hemoglobin levels 75 cases and these cases accounted for 12 deaths $(16 \%)$. A positive rate of increment of deaths with adverse score was obtained but was not found to be statistically significant.

\section{Glasgow coma scale:}

There were 30 cases $(40 \%)$ with low Glasgow coma scale score who were subjected to surgery and accounted for 5 deaths $(16.7 \%)$. There were no patients with score less than 9 in our study. A positive rate of increment of deaths with higher POSSUM score was obtained but was $t$ found to be statistically significant.

Pulse rate:

A total of 5 surgeries $(6.6 \%)$ were done on patients with higher POSSUM scores for pulse rate and accounted for one deaths $(20 \%)$. A positive rate of increment of deaths with higher POSSUM scores was found in our study but was found to be statistically significant.

\section{Blood pressure:}

A total of one procedures (1.3\%) were done on patients with higher POSSUMscore for blood pressure and these cases accounted for one deaths (100\%). A positive rate of increment of deaths with higher POSSUM scores was found in our study group was to be statistically significant.

\section{Respiratory system:}

A total of 30 surgeries (40\%) were performed on patients with higher POSSUM scores and these procedures resulted in 5 deaths $(16.7 \%)$.A positive rate of increment of deaths with higher POSSUM scores for respiratory system was found but was not found to be statistically significant. 


\section{Cardiovascular system:}

There were only20 surgeries (27\%) performed on patients with higher POSSUM scores and resulted in 3 deaths $(15 \%)$. A positive rate of increment of deaths per score was found in our study but was not found to be statistically significant.

Age:

A total of 10 surgeries (13.3\%) were performed on patients with age more than 60years and these cases accounted for 2 deaths $(20 \%)$. A positive rate of increment was found between deaths and higher POSSUM scores for age of the patient.

\section{Multiple surgeries:}

There were 30 multiple surgeries ( 2 surgeries) performed in our study which accounted for 4 deaths. A positive increment of deaths with higher POSSUM score was found.

\section{Discussion}

The basic tenet in medical care has been to provide quality care to the patient to cause reduction in adverse outcome. It is by comparing the adverse outcome rates that we can assess the adequacy of care provided to the patient and evolve new treatment strategies. However, comparison using crude mortality rates can be misleading as it cannot adequately account for the patient"s general condition and the disease process for which he was subjected to surgery. To overcome this shortcoming POSSUM, a risk adjusted scoring system was proposed. In our study we assessed the validity of P-POSSUM in 75 major general surgeries by comparing the observed mortality rate with expected mortality rate. 12 patients died (mortality rates of $15 \%$ (elective) and $16 \%$ (emergency), the total crude mortality rate being 16\%). However on using P-POSSUM the expected mortality rate was 12 deaths. On analysis, there was found to be no statistically significant difference between the observed and expected mortality rates $(\mathrm{P}$ Value $=0.048)$.

An O: E ratio of 0.96 was obtained. Similar findings were obtained by Yii MK and Ng KJ19 (O: E = 1.28), Tekkis15 (O: $\mathrm{E}=0.98)$ and Mohil 20(O: $\mathrm{E}=0.66, \mathrm{x} 2=5.33,9$ d.f., $\mathrm{p}=0.619)$. Hence PPOSSUM was able to accurately predict the adverse outcome following major surgery in our study. On analysing the risk factors we found positive rate of increment with all the risk factors studied but it was not found to be statistically significant with respect to malignancy, total blood loss, serum sodium, blood urea and white cell count. Various factors like decreased immunity and cachexia resulting from malignancy, ischemia and impaired haemostasis resulting from blood loss, uraemia resulting in decreased healing rates, impaired immunity, leucocytosis correlating with the degree of inflammation, toxaemia, hyponatremia resulting into impaired physiological response could be attributed to the effect of these factors on post operative mortality rate.

Therefore adequate and prompt correction can definitely be expected to cause a decrease inadverse outcome rates. Tekkis and others found that total blood loss was not significant enough to alter their statistical analysis in their study but their study predominantly involved elective cases $(26.6 \%)$. Wound infection (20 cases, 26\%) and chest infections (10 cases,13\%) accounted for the majority of complications. Similar results were obtained by Mohil RS (35\% and 20\% respectively)20. Wound infections could be attributed to the large number of patients who had gross peritoneal contamination resulting from hollow visceral perforation resulting in local contamination of the incision site. A raised diaphragm, upper abdominal incision and gross peritoneal contamination resulting into higher rates of chest infections in our group.

\section{Conclusion}

We studied 75 major general surgeries, both elective (26.6\%) and emergency cases (73.3\%), which resulted in 12 deaths ( $16 \%$ mortality rate). On applying P-POSSUM we found that the expected number of deaths for our study group was $13(\mathrm{O}: \mathrm{E}=0.96)$. We found no difference between expected and observed mortality rates. The present study suggests that P-POSSUM is an accurate scoring system for predicting post operative adverse outcome among patients undergoing major general surgeries. The complications of wound infection (26\%) and chest infection (13\%) are a concern and require better care for their prevention following major general surgeries. All the studied risk factors were found to have a positive rate of increment of deaths with higher scores. Presence of malignancy, total blood loss, serum sodium levels and blood urea levels and leukocytosis were found to be significant in our study. Hence adequate and prompt correction of these factors could decrease the mortality rate.This study therefore validates P-POSSUM as a valid means of assessing adequacy of care provided to the patient. P-POSSUM can be used for surgical audit to assess and improve the quality of surgical care and result in better outcome to the patient. 


\section{Summary}

A total of 75 major surgical operations were studied in patients admitted in general surgery department in government Rajaji hospital, Madurai medical college, Madurai. The study group consisted of 20 elective and 55 emergency cases. Duodenal perforation (30 cases), malignancy (3 cases), intestinal obstruction (9 cases), Ileal perforation ( 8 cases), gastric perforation ( 7 cases), appendicular perforations (5cases), limb gangrene (4 cases), obstructed hernia ( 3 cases), others ( 3 cases) were the indications for which the patients were subjected for surgery. Laparotomies accounted for 58 cases, resection anastomosis for 10 cases, amputations for 4 cases and cholecystectomy accounted for 3case. They were scored using P-POSSUM scoring system, physiological scoring was done at the time of admission and operative scoring was done intraoperatively. They were followed up for the first 30 day post operative period for any complications and the outcome was noted. The observed mortality rate was compared with the P- POSSUM expected mortality rate.12 patients died (mortality rates of $15 \%$ (elective) and $16 \%$ (emergency), the total mortality rate of $16 \%$ ) The P-POSSUM expected mortality rate was 13 deaths. An O: E ratio of 0.96 was obtained. There was no statistical difference between the observed and P-POSSUM predicted mortality rates $(\mathrm{p}=0.048)$. On analyzing the risk factors we found positive rate of increment with all the risk factors studied but it was not found to be statistically significant with respect to malignancy, total blood loss, sodium, blood urea, and white cell count, Wound infection (20 cases, 26\%) and chest infections (10 cases, $13 \%$ ) accounted for the majority of complications.

\section{Bibliography}

[1]. Isbister WH, Al Sanea N. POSSUM: a re-evaluation in patients undergoing surgery for rectal cancer. The Physiological and Operative Severity Score for Enumeration of Mortality and Morbidity. ANZ Journal of Surgery 2002;72(6):421-5.

[2]. Shuhaiber JH, Hankins M, Robless P, Whitehead SM. Comparison of POSSUM with P-POSSUM for prediction of mortality in infrarenal abdominal aortic aneurysm repair. Annals of Vascular Surgery 2002;16(6):736-41.

[3]. Tekkis PP, Kessaris N, Kocher HM, Poloniecki JD, Lyttle J, Windsor AC. Evaluation of POSSUM and P-POSSUM scoring systems in patients undergoing colorectal surgery. British Journal of Surgery 003;90(3):340-5.

[4]. Griffiths H, Cuddihy P, Davis S, Parikh S, Tomkinson A. Risk-adjusted comparative audit. Is Possum applicable to head and neck surgery? Clinical Otolaryngology \& Allied Sciences 2002;27(6):517-20.

[5]. Organ N, Morgan T, Venkatesh B, Purdie D. Evaluation of the PPOSSUM mortality prediction algorithm in Australian surgical intensive care unit patients. ANZ Journal of Surgery 2002;72(10):735-8.

[6]. Prytherch DR, Ridler BM, Beard JD, Earnshaw JJ, Audit and Research Committee TVSSoGBaI. A model for national outcome audit in vascular surgery. European Journal of Vascular \& Endovascular Surgery 2001;21(6):477-83.

[7]. Kuhan G, Abidia AF, Wijesinghe LD, Chetter IC, Johnson BF, Wilkinson AR et al. POSSUM and P-POSSUM overpredict mortality for carotid endarterectomy. European Journal of Vascular \& Endovascular Surgery 2002;23(3):209-11.

[8]. Yii MK, Ng KJ. Risk-adjusted surgical audit with the POSSUM scoring system in a developing country. Physiological and Operative Severity Score for the enUmeration of Mortality and morbidity. British Journal of Surgery 2002;89(1):110-3.

[9]. Prytherch DR, Sutton GL, Boyle JR. Portsmouth POSSUM models for abdominal aortic aneurysm surgery.[comment]. British Journal of Surgery 2001;88(7):958-63. 2001;88(7):958-63.

[10]. Prytherch DR, Ridler BM, Beard JD, Earnshaw JJ, The Audit and Research Committee TVSSoGBaI. A model for national outcome audit in vascular surgery. European Journal of Vascular \& Endovascular Surgery 2001;21(6):477-83

[11]. Tekkis PP, Kocher HM, Bentley AJ, Cullen PT, South LM, Trotter GA et al. Operative mortality rates among surgeons: comparison of POSSUM and p-POSSUM scoring systems in gastrointestinal surgery. Diseases of the Colon \& Rectum 1532;43(11):1528-32.

[12]. Irvine CD, Shaw E, Poskitt KR, Whyman MR, Earnshaw JJ, Heather BP. A comparison of the mortality rate after elective repair of aortic aneurysms detected either by screening or incidentally. European Journal of Vascular \& Endovascular Surgery 2000;20(4):374-8.

[13]. Cook SJ, Rocker MD, Jarvis MR, Whiteley MS. Patient outcome alone does not justify the centralisation of vascular services. Annals of the Royal College of Surgeons of England 2000;82(4):268-71.

[14]. Menon KV, Whiteley MS, Burden P, Galland RB. Surgical patients with methicillin resistant staphylococcus aureus infection: an analysis of outcome using P-POSSUM. Journal of the Royal College of Surgeons of Edinburgh 1999;44(3):161-3.

[15]. 15.Midwinter MJ, Tytherleigh M, Ashley S. Estimation of mortality and morbidity risk in vascular surgery using POSSUM and the Portsmouth predictor equation. British Journal of Surgery 1999;86(4):471-4.

[16]. Prytherch DR, Whiteley MS, Higgins B, Weaver PC, Prout WG, Powell SJ. POSSUM and Portsmouth POSSUM for predicting mortality. Physiological and Operative Severity Score for the enUmeration of Mortality and morbidity.[comment]. British Journal of Surgery 1998;85(9):1217-20.

[17]. Wijesinghe LD, Mahmood T, Scott DJ, Berridge DC, Kent PJ, Kester RC. Comparison of POSSUM and the Portsmouth predictor equation for predicting death following vascular surgery.[comment]. British Journal of Surgery 1998;85(2):209-12.

[18]. Mohamed K, Copeland GP, Boot DA, Casserley HC, Shackleford IM, Sherry PG et al. An assessment of the POSSUM system in orthopaedic surgery. Journal of Bone \& Joint Surgery - British Volume 2002;84(5):735-9.

[19]. Sagar PM, Hartley MN, MacFie J, Taylor BA, Copeland GP. Comparison of individual surgeon's performance. Risk-adjusted analysis with POSSUM scoring system. Diseases of the Colon \& Rectum 1996;39(6):654-8.

[20]. Copeland GP, Sagar P, Brennan J, Roberts G, Ward J, Cornford P et al. Risk-adjusted analysis of surgeon performance: a 1-year study.[comment]. British Journal of Surgery 1995;82(3):408-11.

[21]. Copeland GP, Jones D, Wilcox A, Harris PL. Comparative vascular audit using the POSSUM scoring system. Annals of the Royal College of Surgeons of England 1993;75(3):175-7.

[22]. Jones DR, Copeland GP, de Cossart L. Comparison of POSSUM with APACHE II for prediction of outcome from a surgical highdependency unit. British Journal of Surgery 1992;79(12):1293-6.

[23]. Copeland GP, Jones D, Walters M. POSSUM: a scoring system for surgical audit. British Journal of Surgery 1991;78(3):355-60 